\title{
An automated odor test for rats*
}

\author{
STEPHEN A. SHUMAKE $\dagger$, R. DAN THOMPSON \\ and ROGER W. BULLARD \\ Denver Wildlife Research Center \\ Federal Center, Denver, Colorado 80225
}

The odor-testing apparatus described consists of a circular open-field area opening into four glass tubes, each with an odor source at the far end. When a rat is introduced, each tube is blocked by a grid; when the rat has touched each grid (sampled each odor), the tubes automatically open and recording of preference behavior starts. Two measures of preference are recorded by a photocell outside each tube, the number of visits and the time spent near the odor source. A uniformity test with food odor in all tubes showed that the apparatus did not promote position bias. In two separate sensitivity tests, one with food odor and one with estrous female urine odor, male rats significantly preferred an attractive odor in one tube to odorless controls. The apparatus yields relatively sensitive and reliable odor preference determinations by ensuring sampling of each odor before data are recorded and by eliminating visual, auditory and gustatory cues.

A sensitive and reliable test for odor preference is a prerequisite for the study of rodent responses to olfactory stimuli. The study of pheromones and their ultimate isolation and identification often depends on an unbiased, rapid, and dependable behavioral test. Although several reports (Reiff, 1956; Steinbrecher, 1962: Calhoun, 1962; Teichner. 1966: Long \& Tapp, 1967, 1968: Tapp \& Long, 1968: Howard, Palmateer, \& Marsh, 1969: Bronson \& Caroom, 1971) have been published describing tests for odor preference, none has incorporated an apparatus designed to meet all of the following criteria: (1) sensitivity and reliability, (2) olfactory sampling of each odor before preference behavior is recorded, (3) rapid and fully automated data acquisition for individual animals, (4) construction with relatively inert, odor-free materials on exposed surfaces such as glass, stainless steel, and Teflon, ${ }^{1}$ (5) convenient and rapid cleaning after each animal has been tested, (6) no necessity for training or extensive apparatus adaptation. The apparatus described here was designed to meet these criteria and to serve as a practical device for measuring rat odor preference responses to candidate attractants.

*This research was conducted in part with funds provided to the U.S. Bureau of Sport Fisheries and Wildlife by the Agency for International Development under the project "Control of Vertebrate Pests: Rats, Bats, and Noxious Birds." PASA RA(ID) 1-67. The apparatus was constructed under contract for the U.S. Bureau of Sport Fisheries and Wildlife by Life Science Instruments, Inc., Boulder, Colorado. The authors wish to acknowledge the able assistance of C.P. Breidenstein and C. J. Caudill in the design of the apparatus and data collection.

+ Requests for reprints should be sent to Stephen $\mathrm{A}$. Shumake. Denver Wildlife Research Center. Building 16 , Federal Center. Denver, Colorado 80225 .

\section{METHOD \\ Apparatus}

The open-field odor test maze is shown in Fig. 1 (parenthetical numbers in the following description refer to the parts list in the figure). The device is constructed with chemically inert materials, such as stainless steel, glass, and Teflon, that are easily cleaned and relatively odor-free.

The open-field area is Teflon-covered and $2 \mathrm{ft}(61 \mathrm{~cm})$ in diam $x 6.5$ in. $(16.5 \mathrm{~cm})$ in height with a stainless steel grid (7) over the floor. Four glass odor-emission tubes (4) open at right angles into the open-field area; in testing, a 250-ml Erlenmeyer flask (Pyrex No.5100, Stopper No. 8 removed), containing the odor source, is inserted $14 \mathrm{in} .(35.6 \mathrm{~cm})$ into each tube. The flask has six 0.5 -in. $(1.3-\mathrm{cm})$ perforations in the bottom and is bolted to a steel rod and attached to the tube with steel spring bands. A Plexiglas cover (17) is suspended on four steel rollers (6) over the open-field area. Embedded in the periphery of the cover are four gates (11), made of nine 0.5 -in. $(1.3-\mathrm{cm})$ spaced stainless steel rods, that block the entrances of the odor tubes. A small (Dayton Model 2C782) exhaust fan (9), mounted in the center of the cover, slowly draws equal air currents through each tube. Since airflow rate in each of the four odor-emission tubes is too low for measurement with the conventional soap bubble flowmeter technique, indirect effects of flow rates may be determined by inserting into each tube an equal and known weight of volatile fluid (e.g., acetone) with the exhaust fan running. The mean evaporative loss rate in each tube is approximately $285 \%$ faster than in undisturbed air. In cases where differences in flow rate are indicated, adjustable baffles may be fashioned for each tube to limit airflow. Although not shown in Fig. 1. the odorized air from the exhaust fan should be purged from the test room to a window vent with a short length of 2.0 -in. $-(5.1-\mathrm{cm}-)$ diam flexible tubing.

In testing, a single rat is introduced through a hinged cover (8) under the exhaust fan. Dim red light from a $25-\mathrm{W}$ bulb, 18 in. $(45.7 \mathrm{~cm})$ above the cover $(8)$, is the only illumination. As the rat explores the periphery of the open-field area, it makes nose and mouth contact with the stainless steel gates, each of which is connected to a "drink-o-meter" circuit that detects the contact and operates a relay in a control module (not shown in Fig. 1). When the rat has made contact with all four gates, regardless of sequence. a $40-\mathrm{msec}$ pulse from the control module activates a two-way valve (12) connected to an inverted $300-\mathrm{ml}$ glass bottle (10) mounted on the cover: this allows $0.25 \mathrm{ml}$ of a $5.0 \%$ sucrose solution to flow into a drinking fount (13) in the center of the open-field area. When the rat eventually returns to the center and drinks the sucrose solution, a fifth "drink-o-meter" circuit activates a small reversible motor (15) that drives the circular cover $21 \mathrm{deg}$, removing the gates from all four odor tubes. The motor is deactivated by the operation of a microswitch (16). Thus, the rat. after having sampled all four odors and returned to the center of the open field. is now in a free, four-choice situation and can enter any tube for a period of $30 \mathrm{~min}$. A photocell detector $(20)$ is positioned outside each glass tube $4.5 \mathrm{in} .(11.4 \mathrm{~cm})$ from the open-field area so that any light occlusion $0.75 \mathrm{in} .(1.9 \mathrm{~cm})$ into the neck of the odor flask is detected. The photocell light source (not shown in Fig. 1) is located opposite each detector on the outside of each emission tube. Each photocell is connected to a digital counter and an elapsed-time meter. which automatically record the number of times the rat visits the odor (breaks the photocell beam) and the accumulated time it spends near the odor (total seconds the photocell beam is broken).

After the rat has been tested and removed (usually by forcing 


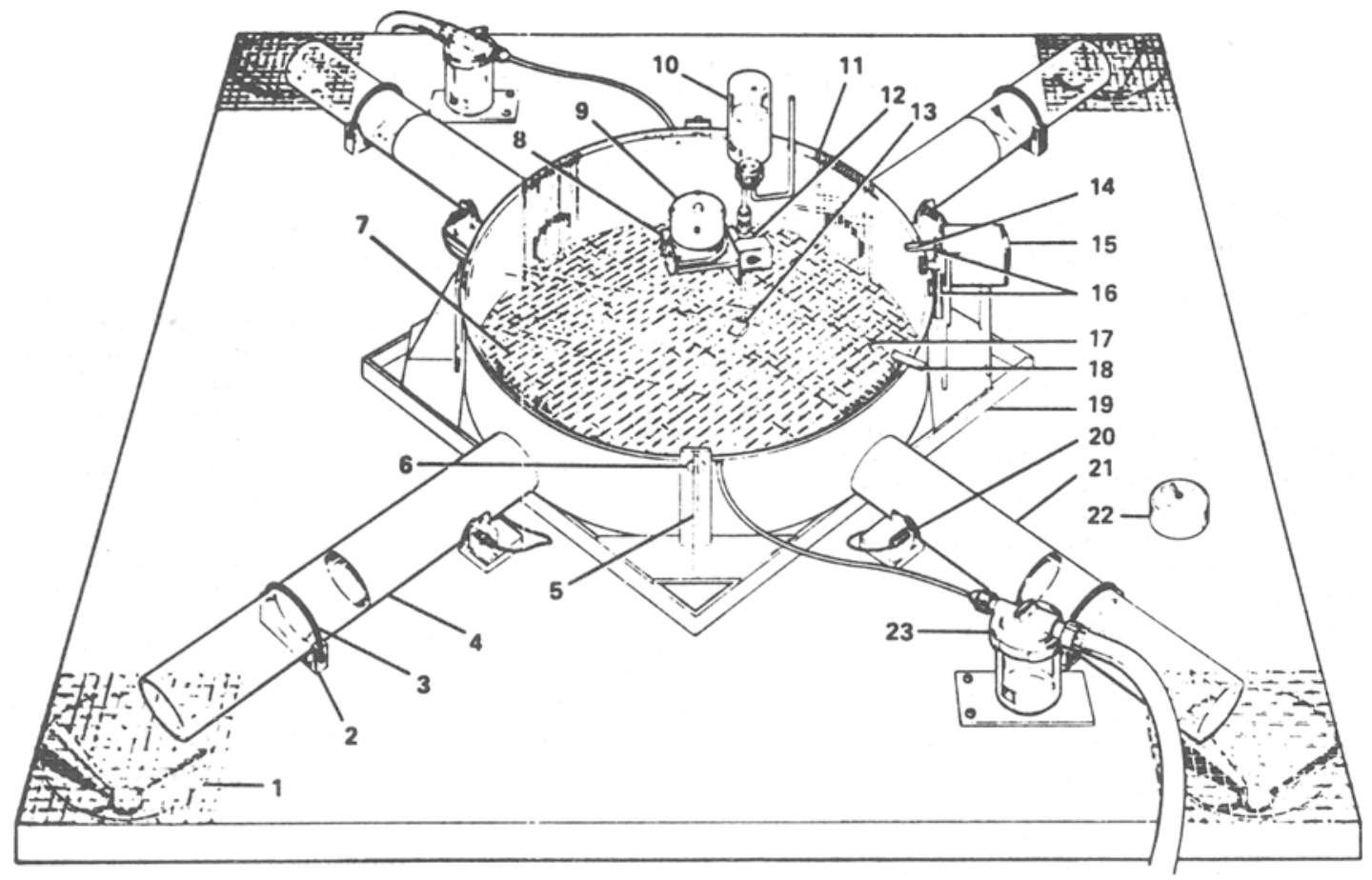

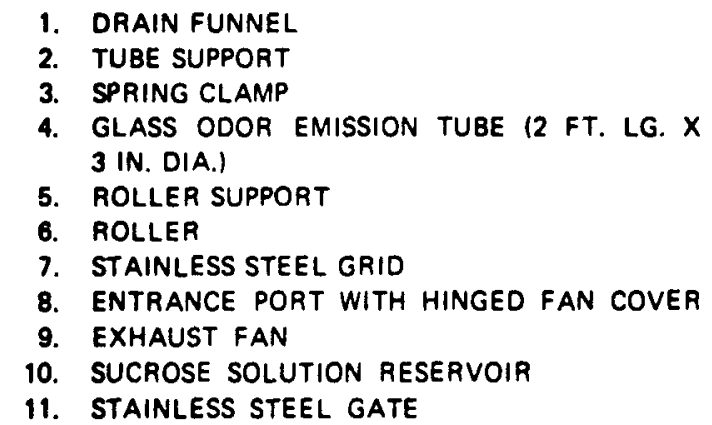

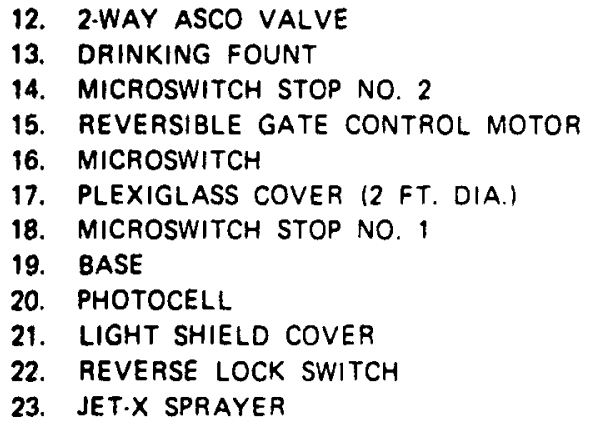

12. 2.WAY ASCO VALVE

13. DRINKING FOUNT

14. MICROSWITCH STOP NO. 2

15. REVERSIBLE GATE CONTROL MOTOR

16. MICROSWITCH

17. PLEXIGLASS COVER (2 FT. DIA.

18. MICROSWITCH STOP NO. 1

19. BASE

20. PHOTOCELL

21. LIGHT SHIELO COVER

23. JET.X SPRAYER

Fig. 1. Diagram of the automated open-field odor test maze.

the animal into one of the emission tubes and capturing it with a transfer cage), a switch (22) is thrown; the motor then reverses to close the gates, and the open-field area and odor emission tubes are automatically cleaned by hot water from two sprayers (23). Excess water is removed at the four drain funnels (1) to a floor drain in the test room. If the next rat is to be introduced immediately, the device is wiped dry with a clean, damp sponge.

\section{Procedure}

Three preliminary tests were conducted with albino rats, Sprague-Dawley strain, to evaluate the apparatus-a uniformity test for position bias, a sensitivity test to determine if rats could reliably detect and respond to an attractant (food odor) in one of the four tubes, and a second sensitivity test with estrous female urine to determine if the apparatus could also be used for attractant pheromones. In all tests, the responses of each rat to each tube were tabulated separately.

\section{Uniformity Test}

Each of the four odor tubes was baited with a mixture of $30 \mathrm{~g}$ of powdered Purina Lab Chow and $25 \mathrm{ml}$ of deionized water. Twenty male rats, about 120 days old, were food deprived for
$12 \mathrm{~h}$ and tested for $30 \mathrm{~min}$ each. To limit rancidity change, the food odor mixture was discarded and replenished after each rat was tested.

\section{Food Odor Localization}

Three tubes were baited with deionized water and the fourth with liquid diet (General Biochemicals No. 166 E.C.); each odor source was a 2-g sterile cotton ball soaked with $30 \mathrm{ml}$ of liquid. Twenty male rats, about 120 days old, that had been fed the liquid diet for 3 days before the trial and food deprived for $12 \mathrm{~h}$, were tested for $30 \mathrm{~min}$ each with fresh liquid diet food odor. The tube to contain the food odor was chosen randomly and equally often for each rat.

\section{Urine Odor Localization}

Three tubes were baited with deionized water and the fourth with pooled urine from four sexually naive, estrous female rats about 120 days old; each odor source was a 2 -g sterile cotton ball soaked with $10 \mathrm{ml}$ of liquid. Twenty sexually experienced males, retired breeders about 300 days old, were tested for 30 min each. The tube to contain the test odor was chosen randomly and equally of ten for each rat. 
Table 1

Comparison of Two Measurements of Odor Preference in Three Tests With 20 Rats Each (Results Analyzed by the Wilcoxon Two-liay Classification Rank-Sum Test (Wilcoxon \& Wilcox, 1964)

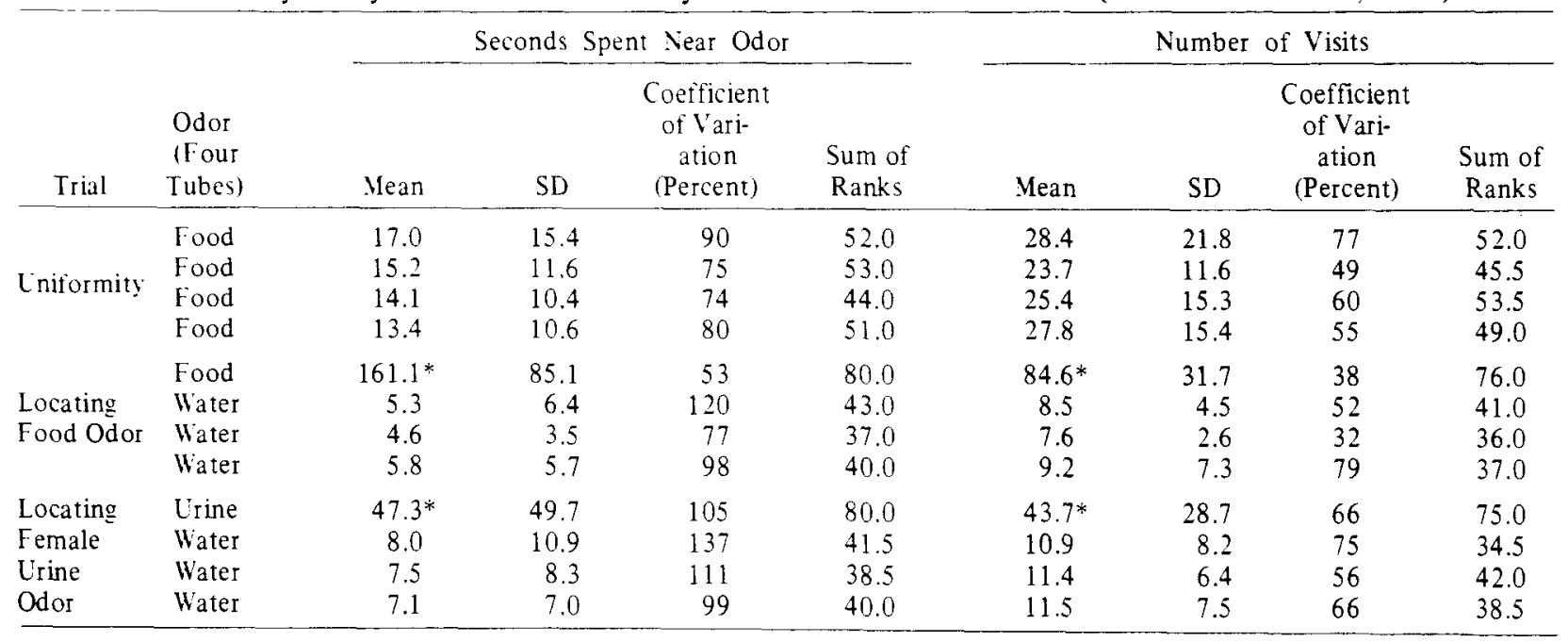

*Significantly different from means for other three tubes (deionized water) at $p<.01$.

\section{RESULTS AND DISCUSSION}

In the uniformity test, both measures of preference-time spent near the odor source and number of visits to it-indicated that there were no sources of bias in the test apparatus or in the test room that would lead to an erroneous assessment of attractant quality. No statistically significant differences appeared in a comparison test of all possible pairs of rank sums for either the elapsed time scores or visitation frequency scores (Table 1).

In the second and third tests, both measures showed that the rats significantly preferred food odor or estrous female urine odor to odorless controls (Table 1). In both tests, all animals spent more time near the test odor than they did near the controls. In the second test, all 20 rats visited the food odor more frequently than they did the controls: in the third test, 19 of the 20 rats visited the urine odor more frequently. Correlation coefficients between the two measures were 0.838 for the second test and 0.924 for the third.

Although the second and third tests showed that rats distinguished an attractive odor and responded to it by longer and more frequent visits, the high coefficients of variation for both measures (Table 1 ) suggest that inter $-\mathrm{S}$ variability is too great to allow adequate tests of preference with weak attractants and small numbers of animals. In some instances with the elapsed time measure, the standard deviation values exceeded the corresponding mean values, yielding coefficients of variation above $100 \%$. Therefore. odor preference evaluations requiring refined discrimination among odors would probably require 30 to 40 rats, each given the 30-min test.

Of the two measures of preference, number of visits appears to be more reliable. since all 12 coefficients of variation for the elapsed time measure exceeded the corresponding coefficients for number of visits (Table 1). However, elapsed time appears to be the more sensitive measure, since the average differences between the test odor and the controls in the second and third tests were about 4-fold for elapsed time (155.9 vs $39.7 \mathrm{sec}$ ). but only about 2.5 -fold for number of visits (76.1 vs 32.5). A weighted sum of these two measures might produce an improved estimate of attractancy, and coefficients that would minimize variability and maximize sensitivity could be computer-calculated by multiple regression.

A potential source of response bias in the automated odor test procedure is the fact that each rat received the $5.0 \%$ sucrose solution after contacting one of the stainless steel gates. It would be possible to produce adventitious chaining between the rat's last gate contact response and sucrose delivery, as indicated by a tendency to repeatedly return to the same gate or tube on subsequent tests. However, this possibility is empirically rather remote. since correlations between the odor position last sampled before sucrose delivery and the final odor position preference were $-0.045,-0.003$. and -0.015 for three replications on 15 food-deprived Ss run in a later separate uniformity test. If, on a given subsequent odor test, the rats made first contact with the gate immediately sampled before sucrose delivery on the previous 30 -min test. sucrose delivery on this test would be delayed until the other three gates had been sampled. breaking up any adventitious chaining effect for the sampling response to a particular odor position. In later tests, we have found that deionized water is as effective as the $5.0 \%$ sucrose solution with nonwater-deprived rats.

The test apparatus offers several improvements over previously used methods. Its design is unique among odor-preference test methods (Teichner. 1966: Long \& 
Tapp. 1968: Howard et al, 1969) in its provision that the $S$ sample all test odors before choice behavior is permitted and recorded. Such prechoice sampling was one of the design criteria considered important by Young and Kappauf (1962) in taste-preference tests with rats and liquid media. Prechoice sampling reduces bias by removing some of the novelty effect of the stimulus and minimizing strictly exploratory responses.

The apparatus permits testing of up to four odors at once, which allows relatively complex comparative tests and faster testing of odors being screened for gross activity. One limitation of the design is that precise control of the location and strength of the odor stimuli is not possible. Odors may tend to become mixed in the open-field area, and simultaneous testing of highly volatile materials may result in poor sensitivity. A four-channel air-dilution olfactometer coupled to the four tubes would help to alleviate some of these problems.

One principal advantage of the apparatus is that no training or orientation period is required, since the design utilizes the typical behavior pattern of rats exposed to an open-field enclosure-that is, to explore the peripheral surfaces. This permits testing of wild as well as domestic rodents. The apparatus virtually eliminates visual, auditory, and gustatory cues associated with test odors, thus minimizing confounding and position bias. The use of inert material, such as Teflon, glass, and stainless steel, along with two hot-water sprayers greatly facilitates cleaning and removal of odor residues between tests. Full automation of both choice presentation and data recording eliminates human interference and variability. The test session is short
(30 min per S), and use of a transfer cage for introducing and removing the animals minimizes handling and associated stress. These features add to the device's usefulness for screening large numbers of odors, such as candidate bait attractants, and for behavioral bioassays to isolate and identify such biologically active compounds as rodent pheromones.

\section{REFERENCES}

Bronson, F. H., \& Caroom, D. Preputial gland of the male mouse; attractant function. Journal of Reproductive Fertility. $1971,25,279-282$.

Calhoun. J. B. The ecology and sociology of the Norway rat. Washington, D.C: Government Printing Office, 1962.

Howard, W. E., Palmateer, S. D., \& Marsh, R. E. A body capacitor-olfactometer for squirrels and rats. Journal of Mammalogy, 1969, 50, 771-776.

Long, C. J., \& Tapp, J. T. Reinforcing properties of odors for the albino rat. Psychonomic Science, 1967, 7, 17-18.

Long, C. J., \& Tapp, J. T. An apparatus for the assessment of the reinforcing properties of odors in small animals. Journal of the Experimental Analysis of Behavior, $1968,11,49.51$.

Reiff. M. Untersuchungen über natüliche und synthetishe Geruchstouffe, die bein Ratten und Mäusen eine stimulierend, Wirbung auslösen. Acta Tropica, 1956, 13, 289-318.

Steinbrecher. W. Die Duftwahl von Wander- und Hausratten. Zeitschrift für Angew andte Zoologie, 1962, 49, 301-349.

Tapp, J. T., \& Long, C. J. A comparison of the reinforcing properties of stimulus onset for several sense modalities. Canadian Journal of Psychology, 1968, 22, 449-455.

Teichner, $W$. H. A method for studying olfaction in the unrestrained rat. Journal of Psychology, 1966, 63, 291-297.

Wilcoxon, F., \& Wilcox, R. A. Some rapid approximate statistical procedures. Peal River, N.Y: Lederle Laboratories, 1964.

Young, P. T., \& Kappauf, W. E. Apparatus and procedures for studying taste preferences in the white rat. American Journal of Psychology, 1962, 75, 482-484.

\section{NOTE}

1. Reference to trade names does not imply endorsement of commercial products by the Federal Government.

(Received for publication January 11, 1973; revision received March 9, 1973.) 